DOI: $10.15393 /$ j3.art.2019.5790

UDC 517.51

M. Rostamian Delavar, S. S. Dragomir, M. De La Sen

\title{
A NOTE ON CHARACTERIZATION OF $h$-CONVEX FUNCTIONS VIA HERMITE-HADAMARD TYPE INEQUALITY
}

\begin{abstract}
A characterization of $h$-convex function via HermiteHadamard inequality related to the $h$-convex functions is investigated. In fact it is determined that under what conditions a function is $h$-convex, if it satisfies the $h$-convex version of HermiteHadamard inequality.
\end{abstract}

Key words: $h$-convex function, Hermite-Hadamard inequality

2010 Mathematical Subject Classification: 26A51, 26D15, $52 A 01$

1. Introduction. The following result is well-known in the literature:

Theorem 1. [6] A function $f:(a, b) \subset \mathbb{R} \rightarrow \mathbb{R}$ is convex if and only if

$$
f\left(\frac{x+y}{2}\right) \leqslant \frac{1}{y-x} \int_{a}^{b} f(t) d t \leqslant \frac{f(x)+f(y)}{2}
$$

holds for all $x, y \in(a, b)$ with $x \neq y$.

Inequality (1) is known as the Hermite-Hadamard integral inequality for convex functions. Note that the left-hand part and the right-hand part of (1) separately are equivalent to the convexity of $f$ (see $[5,6]$ ).

In 2006, the concept of $h$-convex functions related to the nonnegative real functions has been introduced in [9] by S. Varošanec. This class includes a large class of nonnegative functions, such as nonnegative convex functions, Godunova-Levin functions [3], s-convex functions in the second sense [1], and P-functions [2]. In [4], A. Házy used the following definition of $h$-convex functions, which is a generalization of convexexity:

(c) Petrozavodsk State University, 2019 
Definition 1. Let $h:[0,1] \rightarrow \mathbb{R}$ be a function, such that $h \not \equiv 0$. We say that $f:(a, b) \rightarrow \mathbb{R}$ is an $h$-convex function, if for all $x, y \in(a, b)$, $\lambda \in[0,1]$, we have

$$
f(\lambda x+(1-\lambda) y) \leqslant h(\lambda) f(x)+h(1-\lambda) f(y) .
$$

We use this definition for the real functions defined on open intervals $(a, b) \subseteq \mathbb{R}$ in this paper. The $h$-convex version of the Hermite-Hadamard inequality was introduced in [8] by Sarikaya et al. as the following:

Theorem 2. Let $f: I \rightarrow[0, \infty]$ be an integrable $h$-convex function. If $a, b \in I$, with $a<b$, then

$$
\frac{1}{2 h\left(\frac{1}{2}\right)} f\left(\frac{a+b}{2}\right) \leqslant \frac{1}{b-a} \int_{a}^{b} f(x) d x \leqslant[f(a)+f(b)]\left(\int_{0}^{1} h(t) d t\right) .
$$

Motivated by the abovementioned works and results, we, in this paper, reply to the problem of conditions $h$-convexity of a function that satisfies (3). Since inequality (3) is double, we separate the problem to the righthand and the left-hand versions, for the sake of convenience.

2. Main results. To achieve our main results about the characterization of an $h$-convex function via (3), we introduce a primary definition along with an example and then establish a basic lemma related to $h$ convex functions.

Definition 2. A function $h:[0,1] \rightarrow \mathbb{R}$ is said to be self-concave if

$$
h(z x+(1-z) y) \geqslant h(z) h(x)+h(1-z) h(y),
$$

for all $z \in(0,1)$ and $x, y \in[0,1]$.

We can find some simple functions that are self concave.

Example. Consider the function $h(x)=x^{n}$ for $n \in \mathbb{N}$ and $x \in[0,1]$. It is not hard to see that this function is self-concave. In fact, since the function $h$ is nonnegative,

$$
\begin{gathered}
h(\lambda x+(1-\lambda) y)=(\lambda x+(1-\lambda) y)^{n}=\sum_{i=0}^{n}\left(\begin{array}{c}
n \\
i
\end{array}\right)(\lambda x)^{n-i}((1-\lambda) y)^{i} \geqslant \\
\geqslant\left(\begin{array}{l}
n \\
0
\end{array}\right)(\lambda x)^{n}+\left(\begin{array}{l}
n \\
n
\end{array}\right)((1-\lambda) y)^{n}=h(\lambda) h(x)+h(1-\lambda) h(y) .
\end{gathered}
$$

Now consider the function $h(x)=\tan (x)$, for $x \in(0,1)$ and $z \in(0,1)$. Expanding this function and using the self-concavity of $x^{n}$ for $n \in \mathbb{N}$ and 
$x \in[0,1]$, we get

$$
\begin{gathered}
\tan (\lambda x+(1-\lambda) y)=(\lambda x+(1-\lambda) y)+\frac{1}{3}(\lambda x+(1-\lambda) y)^{3}+ \\
+\frac{2}{15}(\lambda x+(1-\lambda) y)^{5}+\frac{17}{315}(\lambda x+(1-\lambda) y)^{7}+\frac{62}{2835}(\lambda x+(1-\lambda) y)^{9}+\ldots \geqslant \\
\geqslant \lambda x+\frac{1}{3}(\lambda x)^{3}+\frac{2}{15}(\lambda x)^{5}+\frac{17}{315}(\lambda x)^{7}+\frac{62}{2835}(\lambda x)^{9}+\cdots+((1-\lambda) y) \\
+\frac{1}{3}((1-\lambda) y)^{3}+\frac{2}{15}((1-\lambda) y)^{5}+\frac{17}{315}((1-\lambda) y)^{7}+\frac{62}{2835}((1-\lambda) y)^{9}+\cdots= \\
=\tan (\lambda x)+\tan ((1-\lambda) y)>\tan (\lambda) \tan (x)+\tan (1-\lambda) \tan (y),
\end{gathered}
$$

which implies the self-concavity of $h(x)=\tan (x)$ on $(0,1)$. Note that we have used the fact that $\tan (x y)>\tan (x) \tan (y)$ for all $x, y \in(0,1)$.

The following lemma plays an important role in obtaining our expected results.

Lemma 1. Let $f:(a, b) \rightarrow \mathbb{R}$ be a continuous function and $h:[0,1] \rightarrow \mathbb{R}$ be a continuous self-concave function. Suppose that for any $x, y \in(a, b)$ with $x \neq y$ there is a $\lambda \in(0,1)$ such that $f(\lambda x+(1-\lambda) y) \leqslant h(\lambda) f(x)+$ $+h(1-\lambda) f(y)$. Then $f$ is $h$-convex on $(a, b)$.

Proof. Without loss of generality, consider $x, y \in(a, b)$ with $x<y$. Define

$$
M_{x, y}=\{\lambda \in[0,1] ; f(\lambda x+(1-\lambda) y) \leqslant h(\lambda) f(x)+h(1-\lambda) f(y)\} .
$$

It is obvious that $M_{x, y}$ is nonempty. Since $f$ and $h$ are continuous on their domains, $M_{x, y}$ is closed in $[0,1]$. We prove that $M_{x, y}=[0,1]$. On the contrary, suppose that $M_{x, y}$ is a proper subset of $[0,1]$; then we can find $\alpha, \beta \in M_{x, y}$ such that $(\alpha, \beta) \subset[0,1] \backslash M_{x, y}$. Set

$$
w=\alpha x+(1-\alpha) y, z=\beta x+(1-\beta) y .
$$

From the assumption, there is a $\lambda \in(0,1)$ such that

$$
f(\lambda w+(1-\lambda) z) \leqslant h(\lambda) f(w)+h(1-\lambda) f(z) .
$$

Also

$$
\left\{\begin{array}{l}
f(w)=f(\alpha x+(1-\alpha) y) \leqslant h(\alpha) f(x)+h(1-\alpha) f(y) \\
f(z)=f(\beta x+(1-\beta) y) \leqslant h(\beta) f(x)+h(1-\beta) f(y) .
\end{array}\right.
$$


Set $t=\lambda \alpha+(1-\lambda) \beta$. It is clear that $t \in(\alpha, \beta)$ and $t \notin M_{x, y}$. Therefore, from the self-concavity of $h$ and relations (4)-(6), we have

$$
\begin{gathered}
f(t x+(1-t) y)>h(t) f(x)+h(1-t) f(y)= \\
=h(\lambda \alpha+(1-\lambda) \beta) f(x)+h(1-(\lambda \alpha+(1-\lambda) \beta)) f(y)= \\
=h(\lambda \alpha+(1-\lambda) \beta) f(x)+h(\lambda(1-\alpha)+(1-\lambda)(1-\beta)) f(y) \geqslant \\
\geqslant[h(\lambda) h(\alpha)+h(1-\lambda) h(\beta)] f(x)+[h(\lambda) h(1-\alpha)+h(1-\lambda) h(1-\beta)] f(y)= \\
=h(\lambda)[h(\alpha) f(x)+h(1-\alpha) f(y)]+h(1-\lambda)[h(\beta) f(x)+h(1-\beta) f(y)] \geqslant \\
\geqslant h(\lambda) f(w)+h(1-\lambda) f(z) \geqslant f(\lambda w+(1-\lambda) z) .
\end{gathered}
$$

On the other hand,

$$
\begin{gathered}
\lambda w+(1-\lambda) z=\lambda(\alpha x+(1-\alpha) y)+(1-\lambda)(\beta x+(1-\beta) y)= \\
=[\lambda \alpha+(1-\lambda) \beta] x+[\lambda(1-\alpha)+(1-\lambda)(1-\beta)] y= \\
=[\lambda \alpha+(1-\lambda) \beta] x+[1-(\lambda \alpha+(1-\lambda) \beta)] y=t x+(1-t) y .
\end{gathered}
$$

So,

$$
f(t x+(1-t) y)=f(\lambda w+(1-\lambda) z)<f(t x+(1-t) y)
$$

which is a contradiction. It follows that $M_{x, y}$ is not a proper subset of $[0,1]$ and hence $M_{x, y}=[0,1]$. Since this happens for any $x, y \in(a, b)$ with $x<y$, we conclude that $f$ is $h$-convex on $(a, b)$.

Theorem 3. Let $f:(a, b) \rightarrow \mathbb{R}$ be a continuous function. Also suppose that $h:[0,1] \rightarrow \mathbb{R}$ is a continuous self-concave function, such that

$$
\frac{1}{y-x} \int_{x}^{y} f(t) d t \leqslant[f(x)+f(y)]\left(\int_{0}^{1} h(t) d t\right),
$$

for all $x, y \in(a, b)$ with $x \neq y$. Then $f$ is $h$-convex on $(a, b)$.

Proof. Suppose that $f$ is not $h$-convex on $(a, b)$. Then, by Lemma 1 , there are $x, y \in(a, b)$ with $x<y$ such that

$$
f(t x+(1-t) y)>h(t) f(x)+h(1-t) f(y) \quad \forall t \in(0,1) .
$$


For such $x$ and $y$,

$$
\begin{aligned}
& \frac{1}{y-x} \int_{x}^{y} f(t) d t=\int_{0}^{1} f(t x+(1-t) y) d t>\int_{0}^{1}[h(t) f(x)+h(1-t) f(y)] d t= \\
& =\left(\int_{0}^{1} h(t) d t\right) f(x)+\left(\int_{0}^{1} h(1-t) d t\right) f(y)=[f(x)+f(y)]\left(\int_{0}^{1} h(t) d t\right) .
\end{aligned}
$$

This is a contradiction. Hence, $f$ is $h$-convex on $(a, b)$.

The following lemma, along with Lemma 1, are the base for characterization of a $h$-convex function via the left-hand side of (3).

Lemma 2. (Also see Theorem 1.1.4 in [5].) Suppose that $\varphi:[a, b] \rightarrow \mathbb{R}$ is a continuous function such that $\varphi(a)=\varphi(b)=0$ and $\varphi(t)>0$ for some $t \in(a, b)$. Then there exists an $x \in(a, b)$ such that

$$
\varphi(x)=\max _{a \leqslant y \leqslant b} \varphi(y) \text { and } \varphi(x)>\varphi(y) \text { for all } a \leqslant y<x .
$$

Proof. From Theorem 4.16 in [7], $\varphi$ attains its maximum $\alpha$ in $[a, b]$. From the assumption, we have $\alpha \geqslant \varphi(t)>0$. Set $M=\{y \in[a, b] ; \varphi(y)=\alpha\}$. Since $\varphi$ is continuous, $M$ is a nonempty compact subset of $[a, b]$, such that $a, b \notin M$. If we put $x=\inf \{y ; y \in M\}$, then

$$
\varphi(x)=\alpha=\max _{a \leqslant y \leqslant b} \varphi(y),
$$

and $f(y)<f(x)$ for all $a \leqslant y<x$.

In what follows, we assume that the function $h:[0,1] \rightarrow \mathbb{R}$ satisfies the conditions

$$
\left\{\begin{array}{l}
h(\lambda)+h(1-\lambda)=1 \text { for all } \lambda \in(0,1), \\
h(0)=0
\end{array}\right.
$$

Lemma 3. Let $h:[0,1] \rightarrow \mathbb{R}$ be a continuous self-concave function. Suppose that $f:(a, b) \rightarrow \mathbb{R}$ is a continuous function and for any $x \in(a, b)$, $\varepsilon>0$, there exist $y, z \in(a, b) \cap(x-\varepsilon, x+\varepsilon)$ with $y<x<z$ such that

$$
f(x)=f(\lambda y+(1-\lambda) z) \leqslant h(\lambda) f(y)+h(1-\lambda) f(z) \text { for some } \lambda \in(0,1) .
$$

Then $f$ is $h$-convex on $(a, b)$. 
Proof. If $f$ is not $h$-convex, then by Lemma 1 , there are $x_{1}, x_{2} \in(a, b)$ with $x_{1} \neq x_{2}$ (assume that $x_{1}<x_{2}$ ) such that

$$
f\left(\lambda x_{1}+(1-\lambda) x_{2}\right)>h(\lambda) f\left(x_{1}\right)+h(1-\lambda) f\left(x_{2}\right) \text { for all } \lambda \in(0,1) .
$$

Consider the function $g:\left[x_{1}, x_{2}\right] \rightarrow \mathbb{R}$ defined by

$$
\begin{gathered}
g(y)=g\left(\lambda x_{1}+(1-\lambda) x_{2}\right)= \\
=f\left(\lambda x_{1}+(1-\lambda) x_{2}\right)-f\left(x_{1}\right)-\frac{f\left(x_{2}\right)-f\left(x_{1}\right)}{x_{2}-x_{1}}\left(h(\lambda) x_{1}+h(1-\lambda) x_{2}-x_{1}\right) .
\end{gathered}
$$

It is clear that $g$ is continuous on $\left[x_{1}, x_{2}\right]$ and $g\left(x_{1}\right)=g\left(x_{2}\right)=0$. Also, from (7) and (8), we get

$$
\begin{gathered}
g\left(\lambda x_{1}+(1-\lambda) x_{2}\right)=f\left(\lambda x_{1}+(1-\lambda) x_{2}\right)-f\left(x_{1}\right)- \\
-\frac{f\left(x_{2}\right)-f\left(x_{1}\right)}{x_{2}-x_{1}}\left((1-h(\lambda)) x_{2}-(1-h(\lambda)) x_{1}\right)= \\
=f\left(\lambda x_{1}+(1-\lambda) x_{2}\right)-h(\lambda) f\left(x_{1}\right)-h(1-\lambda) f\left(x_{2}\right)>0 .
\end{gathered}
$$

Lemma 2 and (9) imply that there is an $x \in\left(x_{1}, x_{2}\right)$ such that

$$
g(x)=\max _{x_{1} \leqslant y \leqslant x_{2}} g(y) \text { and } g(x)>g(y) \text { for } x_{1} \leqslant y<x .
$$

Hence, $x=t x_{1}+(1-t) x_{2}$ for some $0<t<1$. Now choose $x_{0}, y_{0} \in\left[x_{1}, x_{2}\right]$ such that $x_{1} \leqslant x_{0}<x<y_{0} \leqslant x_{2}$. Therefore, from (10) for any $\lambda \in(0,1)$,

$$
\begin{aligned}
g(x) & =[h(\lambda)+h(1-\lambda)] g(x)>h(\lambda) g\left(x_{0}\right)+h(1-\lambda) g\left(y_{0}\right) . \\
f(x)- & f\left(x_{1}\right)-\frac{f\left(x_{2}\right)-f\left(x_{1}\right)}{x_{2}-x_{1}}\left(h(\lambda) x_{0}+h(1-\lambda) y_{0}-x_{1}\right)> \\
& >h(\lambda)\left[f\left(x_{0}\right)-f\left(x_{1}\right)-\frac{f\left(x_{2}\right)-f\left(x_{1}\right)}{x_{2}-x_{1}}\left(x_{0}-x_{1}\right)\right]+ \\
& +h(1-\lambda)\left[f\left(y_{0}\right)-f\left(x_{1}\right)-\frac{f\left(x_{2}\right)-f\left(x_{1}\right)}{x_{2}-x_{1}}\left(y_{0}-x_{1}\right)\right] .
\end{aligned}
$$

From (7) we deduce, simplifying (12):

$$
f(x)>h(\lambda) f\left(x_{0}\right)+h(1-\lambda) f\left(y_{0}\right) \text { for all } \lambda \in(0,1) .
$$


Since $x_{0}, y_{0}$, and $\lambda$ are arbitrary, (13) contradicts the assumption. Hence, $f$ is an $h$-convex function on $(a, b)$.

Using Lemma 3, as an immediate consequence we have two following lemmas. For more details about this kind of results related to the convex functions, see [6].

Corollary 1. Let $h:[0,1] \rightarrow \mathbb{R}$ be a continuous self-concave function. Suppose that $f:(a, b) \rightarrow \mathbb{R}$ is a continuous function and for any $x \in(a, b)$, $\varepsilon>0$, there exists a $\delta \in(0, \varepsilon)$ such that

$$
f(x) \leqslant h(1 / 2)[f(x-\delta)+f(x+\delta)] .
$$

Then $f$ is $h$-convex on $(a, b)$.

Proof. In Lemma 3, take $y=x-\delta, z=x+\delta$ and $\lambda=1 / 2$.

Lemma 4. Let $h:[0,1] \rightarrow \mathbb{R}$ be a continuous self-concave function. Suppose that $f:(a, b) \rightarrow \mathbb{R}$ is a continuous function and for any $x \in(a, b)$, $\varepsilon>0$, there exists $\delta \in(0, \varepsilon)$ such that

$$
f(x) \leqslant \frac{h(1 / 2)}{\delta} \int_{x-\delta}^{x+\delta} f(u) d u .
$$

Then $f$ is $h$-convex on $(a, b)$.

Proof. Suppose that $f$ is not $h$-convex on $(a, b)$. From Corollary 1 , there are $x \in(a, b)$ and $\varepsilon>0$ such that $a<x-\varepsilon<x+\varepsilon<b$ and

$$
f(x)>h(1 / 2)[f(x-\delta)+f(x+\delta)] \text { for any } 0<\delta<\varepsilon .
$$

Integrating with respect to $\delta$ in the above inequality, we get

$$
\begin{aligned}
& \frac{1}{h(1 / 2)} \int_{0}^{\delta} f(x) d t>\int_{0}^{\delta} f(x-t) d t+\int_{0}^{\delta} f(x+t) d t= \\
& =-\int_{x}^{x-\delta} f(u) d u+\int_{x}^{x+\delta} f(u) d u=\int_{x-\delta}^{x+\delta} f(u) d u .
\end{aligned}
$$

So,

$$
f(x) \cdot \delta \leqslant h(1 / 2) \int_{x-\delta}^{x+\delta} f(u) d u .
$$


This contradicts the assumption and, hence, $f$ is $h$-convex on $(a, b)$.

Now, using Lemma 4, we can obtain a characterization-type theorem for $h$-convex functions via the left-hand side of (3).

Theorem 4. Let $h:[0,1] \rightarrow \mathbb{R}$ be a continuous self-concave function. Suppose that $f:(a, b) \rightarrow \mathbb{R}$ is a continuous function and for all $y, z \in(a, b)$ with $y \neq z$ we have

$$
\frac{1}{2 h(1 / 2)} f\left(\frac{y+z}{2}\right) \leqslant \frac{1}{z-y} \int_{y}^{z} f(u) d u
$$

then $f$ is $h$-convex on $(a, b)$.

Proof. Suppose that $f$ is not $h$-convex on $(a, b)$. From Lemma 4 , there exist $x \in(a, b)$ and $\varepsilon>0$ such that for all $\delta \in(0, \varepsilon)$

$$
f(x)>\frac{h(1 / 2)}{\delta} \int_{x-\delta}^{x+\delta} f(u) d u .
$$

Now, if we choose $\delta<\varepsilon$ and $y, z \in(a, b)$ with $y<z$ such that

$$
\left\{\begin{array}{l}
x=\frac{1}{2} y+\frac{1}{2} z \\
x-y=z-x=\delta
\end{array}\right.
$$

then we have

$$
f\left(\frac{y+z}{2}\right)>\frac{2 h(1 / 2)}{z-y} \int_{y}^{z} f(u) d u .
$$

This contradicts (14). Thus, $f$ is $h$-convex on $(a, b)$.

\section{References}

[1] Breckner W. W. Stetigkeitsaussagen für eine Klasse verallgemeinerter konvexer funktionen in topologischen linearen Räumen. Publ. Inst. Math., 1978, vol. 23, pp. 13-20.

[2] Dragomir S. S., Pečarić J., Persson L. E. Some inequalities of Hadamard type. Soochow J. Math., 1995, vol. 21, pp. 335-341.

[3] Godunova E. K., Levin V. I. Neravenstva dlja funkcii širokogo klassa, soderžaščego vypuklye, monotonnye $i$ neko-torye drugie vidy funkcii. Vyčislitel. Mat. i. Mat. Fiz. Mežvuzov. Sb. Nauč. Trudov, pp. 138-142, MGPI, Moskva, 1985. 
[4] Házy A. Bernstein-Doetsch type results for h-convex functions. Math. Ineq. Appl., 2011, vol. 14, no. 3, pp. 499-508. DOI: https://doi .org/10.7153/ mia-14-42.

[5] Niculescu C. P., Persson L. E. Convex functions and their applications: a contemporary approach. Springer, Berlin, CMS Books in Mathematics, 2006.

[6] Robert A. W., Varbeg D. E. Convex functions. Academic Press, New York, 1973.

[7] Rudin W., Principles of mathematical analysis. McGraw-Hill, 1976.

[8] Sarikaya M. Z., Saglam A., Yildirim H. On Some Hadamard-Type Inequalities for h-Convex Functions. J. Math. Ineq., 2008, vol. 2, no. 3, pp. $335-$ 341. DOI: https://doi.org/10.7153/jmi-02-30.

[9] Varošanec S. On h-convexity. J. Math. Anal. Appl., 2007, vol. 326, pp. $303-$ 311. DOI: https://doi.org/10.1016/j.jmaa.2006.02.086.

Received December 18, 2018.

In revised form, March 11, 2019.

Accepted March 12, 2019.

Published online April 1, 2019.

\section{Rostamian Delavar}

Department of Mathematics, Faculty of Basic Sciences, University of Bojnord, Bojnord, Iran

E-mail: m.rostamian@ub.ac.ir

S. S. Dragomir

Mathematics, College of Engineering \& Science, Victoria University,

PO Box 14428, Melbourne City, MC 8001, Australia

E-mail: sever.dragomir@vu.edu.au

M. De La Sen

Institute of Research and Development of Processes, University of Basque Country, Campus of Leioa (Bizkaia) - Aptdo. 644 - Bilbao, Bilbao, 48080, Spain

E-mail: manuel.delasen@ehu.eus 